EPJ manuscript No.

(will be inserted by the editor)

\title{
The Electrostatic Persistence Length of Polymers beyond the OSF Limit
}

\author{
Ralf Everaers ${ }^{1}$, Andrey Milchev ${ }^{1,2}$ and Vesselin Yamakovi,2 a \\ 1 Max-Planck-Institut für Polymerforschung, Postfach 3148, D-55021 Mainz, Germany \\ , 2 Institute for Physical Chemistry, Bulgarian Academy of Sciences, G. Bonchev Street, Block 11, 1113 Sofia, Bulgaria \\ the date of receipt and acceptance should be inserted later
}

\begin{abstract}
We use large scale Monte Carlo simulations to test scaling theories for the electrostatic persistence length $l_{e}$ of isolated, uniformly charged polymers with Debye-Hückel intrachain interactions in the limit where the screening length $\kappa^{-1}$ exceeds the intrinsic persistence length of the chains. Our simulations cover a significantly larger part of the parameter space than previous studies. We observe no significant deviations from the prediction $l_{e} \propto \kappa^{-2}$ by Khokhlov and Khachaturian which is based on applying the Odijk-Skolnick-Fixman theory to the stretched de Gennes-Pincus-Velasco-Brochard polyelectrolyte blob chain. A linear or sublinear dependence of the persistence length on the screening length can be ruled out. We argue that previous numerical results pointing into this direction are probably due to a combination of excluded volume and finite chain length effects. The paper emphasizes the role of scaling arguments in the development of useful representations for experimental and simulation data.
\end{abstract}

PACS. 6 4.60.-i -3 6.20.-r -8 7.15.By

\section{Introduction}

The theoretical understanding of macromolecules carrying ionizable groups is far from complete [1,2]. In spite of the long range of the interactions, the systems are often discussed using analogies to neutral polymers. A prominent example is the concept of an electrostatic persistence length, which was introduced more than 20 years ago by Odijk [3] and by Skolnick and Fixman [4] (OSF). They considered a semiflexible polymer or wormlike chain (WLC) with intrinsic persistence length $l_{0}$ and DebyeHückel (DH) screened electrostatic interactions $U_{D H} / k_{B} T=$ $\left(q^{2} l_{B} / r\right) \exp (-\kappa r)$ between charges $q / e$ spaced at regular intervals $A$ along the chain. The Bjerrum length $l_{B}$ characterizes the strength of the electrostatic interactions and is defined as the distance where the Coulomb energy of two unit charges $e$ is equal to $k_{B} T$. Due to the presence of mobile ions the bare Coulomb interaction is cut off beyond the screening length $\kappa^{-1}$. OSF were interested in bending fluctuations and considered the resulting increase of the electrostatic energy relative to the straight ground state. In the ("OSF") limit $\kappa^{-1} \ll l_{0}$ where the screening length is smaller than the intrinsic persistence length of the chain and to lowest order in the local curvature, the Debye-Hückel interaction makes an additive contribution to the bending rigidity. As a consequence, a WLC with

\footnotetext{
a present address: Argonne National Laboratory, Materials Science Division, Build. 212, 9700 S. Cass Avenue, Argonne, IL-60439, USA
}

DH interactions (DHWLC) behaves in this limit on large length scales like an ordinary WLC with renormalized persistence length

$$
\begin{aligned}
& l_{p}=l_{0}+l_{O S F} \\
& l_{O S F}=\frac{q^{2} l_{B}}{4 A^{2} \kappa^{2}}
\end{aligned}
$$

Ever since, there has been a lively debate on how to extend the theory to parameter ranges beyond the OSF limit $\kappa^{-1} \ll l_{0}$. Barrat and Joanny (BJ) [0] have shown that the original OSF derivation breaks down, if the chains start to bend significantly on length scales comparable to the screening length. As a consequence, Eq. (2) cannot simply remain valid beyond the OSF limit as was sometimes speculated [6]. Two main scenarios, which we denote by "OSFKK" and "KMBJ" after the initials of the main authors, have been discussed in the literature:

OSFKK According to Khokhlov and Khachaturian (KK) [7] the OSF theory can be applied to a "stretched chain of polyelectrolyte blobs", a concept introduced by de Gennes et al. [8 to describe the behavior of weakly charged flexible polyelectrolytes in the absence of screening. The persistence length of the blob chain is then calculated from Eq. (2) using suitably renormalized parameters.

KMBJ Refinements by Muthukumar [9, 10, 11] of the original theory of Katchalsky 12] treat electrostatic interactions in strict analogy to short-range excluded vol- 
ume interactions. Quite interestingly, the results are consistent with the scaling picture of de Gennes et al. 8 in the two limits of strong and vanishing screening. Moreover, they are supported by recent calculations by BJ and others [5, 13 who determined the persistence length of the blob chain in a variational procedure and found $l_{e} \sim \kappa^{-1}$.

In addition, there is a number of recent theories which fall into neither of the two classes outlined above [14, 15, 16, 17, 18]. While there is a growing consensus among theoreticians that the OSFKK result is asymptotically correct [19, 20,21, experiments [22, 23, 24, 25,26 as well as computer simulations 27,28,29 have consistently provided evidence for a comparatively weak $\kappa$-dependence of the electrostatic presistence length.

The purpose of the present paper is to shed some new light on this problem by combining a scaling analysis with large scale Monte Carlo simulations. We reexamine the KMBJ and the OSFKK theory in order to extract guidance for the data analysis and the choice of simulation parameters. As a result we are able (i) to rule out the KMBJ theory, (ii) to provide benchmark results for analytical solutions of the DHWLC model as well as (iii) for a comparison to experiments in order to clarify if the systems under consideration are actually described by the DHWLC model or if additional effects such as solvent quality or counter-ion condensation need to be taken into account as well.

The paper is organized as follows: In section 2 we review the predictions of the KMBJ and OSFKK theories, followed by a discussion in section 3 of how experiments and simulations should be set up and analyzed in order to discriminate between the two scaling pictures. Details of our Monte Carlo simulations can be found in in section 4 . We present our results in section 5 and close with a brief discussion.

\section{Scaling theories of intrinsically flexible Debye-Hückel chains}

The DHWLC is characterized by the following set of parameters: $q, l_{B}, \kappa, A, f, l_{0}$, and $L_{t o t}$, where $L_{t o t}$ denotes the total chain length and $f \leq 1$ the fraction of ionized charged groups which needs to be determined independently for experimental systems. Setting $f=l_{B} / A$ is a rough way of accounting for Manning condensation [1] of counter ions in cases where $l_{B}<A$. Correlation functions can be calculated for chain segments with arbitrary contour length $L<L_{t o t}$. We focus on the non-OSF limit $l_{B}, A, l_{0} \ll \kappa^{-1} \ll \sqrt{\left\langle r^{2}\right\rangle\left(L_{t o t}\right)}$ where the screening length is larger than all microscopic length scales of the polymer model but smaller than the size of the entire chain.

For an understanding of the physics, some of these length scales and parameters are less relevant than others. For example, the actual distribution of the charges on the chain should be unimportant as long as $A \ll \kappa^{-1}$. In our simulations we use discrete charges spaced by a distance equal to the intrinsic persistence length, while the scaling arguments assume a continuous charge distribution. Similarly, in the non-OSF limit with $l_{0} \ll \kappa^{-1}$ the physics should not depend on the details of the WLC crossover from rigid rod to random coil behavior for $L \approx l_{0}$. In our simulations we therefore use freely jointed chains (FJC) whose (Kuhn) bond length $b$ corresponds (up to a henceforth neglected factor of two) to the persistence length of a WLC. Finally, it is convenient to consider the limit of infinite total chain length in order to eliminate the $L_{t o t}$ dependence. Again the practical limitation to $N_{t o t}=L_{t o t} / b=$ 4096 segments should be unimportant, since our chains always fulfill the condition $\kappa^{-1} \ll \sqrt{\left\langle r^{2}\right\rangle\left(L_{t o t}\right)}$.

The remaining independent parameters (the line charge density $f q / A, l_{B}, \kappa, l_{0}$ and $\left.L\right)$ can be reduced further using the notion of a "polyelectrolyte blob" which was introduced by de Gennes et al. [8] to describe the crossover from locally unchanged chain statistics to stretching on long length scales.

Consider first weakly charged flexible polyelectrolytes, where the electrostatic interactions are irrelevant on the length scales comparable to the intrinsic persistence length $l_{0}$. On larger length scales an undisturbed WLC with a contour length $L$ has a spatial extension $\left\langle r^{2}\right\rangle=2 L l_{0}$. Neglecting prefactors, the electrostatic energy of such a chain is given by $U_{e} / k_{B} T \simeq q^{2}(f L / A)^{2} l_{B} / \sqrt{\left\langle r^{2}\right\rangle}$. Electrostatic interactions become relevant for $U_{e} / k_{B} T \geq 1$ or chain lengths $L$ exceeding

$$
l_{g}=l_{0}^{1 / 3}\left(\frac{A^{2}}{f^{2} q^{2} l_{B}}\right)^{2 / 3}
$$

and whose spatial extension is given by

$$
\xi=l_{0}^{2 / 3}\left(\frac{A^{2}}{f^{2} q^{2} l_{B}}\right)^{1 / 3} .
$$

However, this derivation breaks down for strongly interacting systems where the contour length per blob becomes smaller than the intrinsic persistence length. In this case, a similar argument can be made for a WLC with $L<l_{0}$ and $\left\langle r^{2}\right\rangle=L^{2}$ yielding

$$
l_{g}=\xi=\frac{A^{2}}{q^{2} f^{2} l_{B}} .
$$

Both definitions match for $l_{g}=l_{0}$, hiding a subtle crossover 20 behind a crudely renormalized system of units. Throughout the paper all quantities will be expressed using these natural units of contour length and spatial distance. On a scaling level, they become a function of only two dimensionless parameters: the reduced chain or segment length

$$
X=L / l_{g}
$$

and the reduced screening length

$$
Y=(\kappa \xi)^{-1}
$$

In the model under consideration the electrostatic interactions are purely repulsive. Therefore the chains are 


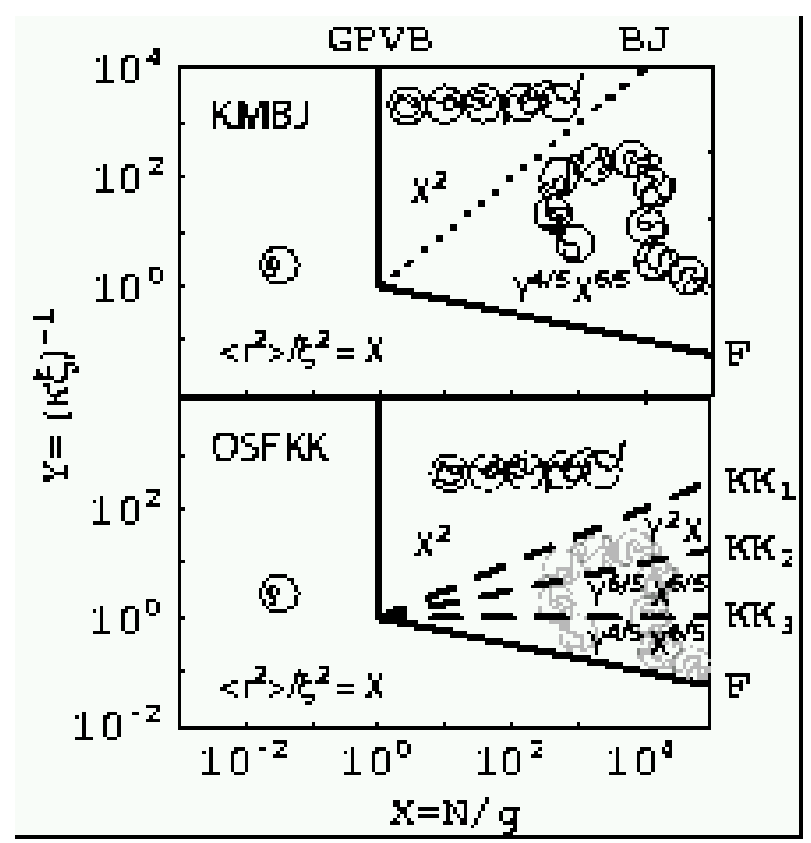

Fig. 1. Conformations of DHWLC beyond the OSF limit as a function of reduced chain length and reduced screening length. Electrostatic interactions are relevant in the upper right part of the map which is limited by solid black lines. The dashed and dotted lines correspond to crossover lines in the OSFKK and KMBJ scaling theories respectively. We have also included the scaling predictions Eqs. (8) to (13) for the chain radii. For details see the main text.

always extended relative to the neutral case. For $\kappa l_{B} \ll$ 1 the details of the process are quite involved and difficult to treat from first principles. The approximation schemes used are often based on mechanical analogies such as stretching, bending and swelling due to short-range excluded volume interactions and have been reviewed in Ref. [1,2]. We have summarized the various predictions in terms of a schematic map of the $X Y$ parameter space (Figure 11). Before we discuss the controversial parts, we first present those aspects which seem well understood:

- The Debye-Hückel interaction is irrelevant inside the electrostatic blob, i.e. for weakly charged chains

$$
\left\langle r^{2}\right\rangle / \xi^{2} \simeq X \quad \text { for } X<1
$$

For strongly charged chains, this regime does not exist.

- In the absence of screening, when the monomers interact via an infinite range Coulomb potential, the chains are stretched into a "blob pole":

$$
\left\langle r^{2}\right\rangle / \xi^{2} \simeq X^{2} \quad \text { for } 1<X<\infty \text { and } Y \rightarrow \infty
$$

In Figure 1 we have marked the line dividing these two regimes as "GPVB" after the initials of the authors of Ref. [8] where the notion of the electrostatic blob was introduced.
- For sufficiently long chains, the Debye-Hückel interaction becomes effectively short-ranged, leading to selfavoiding walk (SAW) behavior

$$
\left\langle r^{2}\right\rangle / \xi^{2} \sim X^{2 \nu} \quad \text { for } 0<Y<\infty \text { and } X \rightarrow \infty
$$

where $\nu \approx 3 / 5$ is the usual Flory exponent.

- For strong screening with $q^{2} l_{B}<\kappa^{-1}<A$ the DebyeHückel interaction reduces to an ordinary excluded volume potential with a second virial coefficient $v \simeq$ $q^{2} l_{B} \kappa^{-2}$ between charges. Using a conventional Flory argument to balance the two-body repulsion $v(f L / A)^{2} / R_{F}^{3}$ with the entropic elasticity of a Gaussian chain $R_{F}^{2} /\left(L l_{0}\right)$, one obtains:

$$
\left\langle r^{2}\right\rangle / \xi^{2} \simeq\left\{\begin{array}{ll}
X & \text { for } X<Y^{-4} \\
Y^{4 / 5} X^{6 / 5} & \text { for } X>Y^{-4}
\end{array} \text { for } Y \ll 1\right.
$$

In Figure 11 the corresponding line, beyond which the short range excluded volume interaction becomes relevant, is marked as "F".

The controversial parts of the phase diagram concern the crossover from the blob pole to the self-avoiding walk regime. The problem is often treated in analogy to a simple WLC. With an Onsager virial coefficient $v \simeq l_{p}^{2} d$ between rigid segments of length $l_{p}$ and diameter $d$, excluded volume effects become relevant beyond a "Flory length" $l_{F}=$ $l_{p}^{3} / d^{2}$ leading to a Flory radius of $R_{F}^{2} \simeq d^{2 / 5} l_{p}^{8 / 5}\left(L / l_{p}\right)^{6 / 5}$. In the case of the blob chain, the diameter of the electrostatically excluded volume is given by $d \simeq \kappa^{-1}$ [6, 30. However, there is disagreement with respect to the $\kappa$-dependence of the electrostatic persistence length $l_{e}$.

- Variational approaches such as the theory of Barrat and Joanny (BJ) often predict $l_{e} \simeq d \simeq \kappa^{-1}$. As a consequence, $l_{F}=l_{p}=\kappa^{-1}$ so that there is a direct crossover from the stiff blob chain to a SAW regime when the contour length $\xi \frac{N}{g}$ of the blob chain reaches the screening length $\kappa^{-1}$. Using dimensionless units this corresponds to $X=N / g=l_{F} / \xi=l_{p} / \xi=1 /(\kappa \xi)=$ $Y$ (the dotted line in Figure 1 marked "BJ"). The result

$$
\left\langle r^{2}\right\rangle / \xi^{2} \simeq Y^{4 / 5} X^{6 / 5} \quad \text { for } X>Y \text { and } Y \gg 1
$$

is identical to Eq. (11). On a scaling level, the predictions of the BJ theory coincide with those of the excluded volume theories of Katchalsky [12] and Muthukumar [9, 10, 11.

- Most theories favour the relation $l_{e} \simeq \xi^{-1} \kappa^{-2}$ first obtained by Khokhlov and Khachaturian (KK). KK argued that the OSF result Eq. (2) should also apply to a stretched chain of blobs with line charge density $f q l_{g} /(A \xi)$ so that $l_{e} / \xi=1 /(\kappa \xi)^{2}=Y^{2}$. Since $l_{e} \gg d$, the resulting phase diagram is considerably more complicated. The blob chain starts to bend for reduced segment lengths $X$ exceeding $l_{p} / \xi=l_{e} / \xi=Y^{2}$ (the 
" $\mathrm{KK}_{1}$ " line in Figure 1), while excluded volume effects become relevant beyond $l_{F} / \xi=1 /(\kappa \xi)^{4}=Y^{4}$ ("KK${ }_{2}$ ").

$$
\left\langle r^{2}\right\rangle / \xi^{2} \simeq\left\{\begin{array}{ll}
Y^{2} X & \text { for } Y^{2}<X<Y^{4} \\
Y^{6 / 5} X^{6 / 5} & \text { for } X>Y^{4}
\end{array} \text { for } Y \gg 1\right.
$$

Finally, and in contrast to the KMBJ theory, the OSFKK approach implies another crossover ("KK 3 ") within the SAW regime at $Y=1$ from Eq. (13) to Eq. (11).

\section{Implications for Data Production and Analysis}

In general, scaling theories make two kinds of predictions: (i) about the existence of characteristic length scales or crossover lines in conformation space and (ii) about the asymptotic behavior of observables in the areas between these crossover lines. In principle, attempts at refutation can aim at either type of prediction. However, in the present case the identification of asymptotic exponents turns out to be particularly difficult. Apart from numerical prefactors and logarithmic corrections one is faced with four problems:

- Although the various regimes predicted by the KMBJ and the OSFKK theory are characterized by different combinations of powers of $X$ and $Y$, the exponents are often similar and the absolute differences between the predicted chain extensions relatively small.

- At least on a scaling level all crossover lines meet at $X=Y=1$ for chain and screening length of the order of the diameter of the polyelectrolyte blob. In the case of the OSFKK theory some of the predicted regimes are extremely narrow in the sense that chain lengths of $X=10^{4}$ blobs are required for a width of one order of magnitude in $Y$-direction. This validity range would seem to be the absolute minimum for identifying power law behavior.

\footnotetext{
1 For example, in the absence of screening the long-range Coulomb interactions along the blob pole create a tension which grows logarithmically with the chain length $N$. As a consequence, the blob diameter is reduced and the chains grow with $R_{g} \sim N \log ^{1 / 3}(N)$. In particular, a $N$ monomer segment of a longer chain will always be more extended than a $N$ monomer chain whereby the deformation is strongest for segments located near the center of the longer polymer 8, 31. In the presence of screening, this effect leads on the one hand to an increase of the contour length of the blob chain. On the other hand, the correspondingly reduced line charge density results in a reduction of the OSFKK persistence length. Similarly, but neglecting the stretching, there are logarithmic corrections to the electrostatically excluded volume around the blob chain 30. Moreover, although fairly robust, the Flory argument used to estimate the excluded volume effects is far from exact. In addition to the aforementioned crossovers, a complete theory will have to account for all of these effects.
}

- The crossover lines are neither parallel to each other nor to the "natural" $X$ and $Y$ directions of variations of chain and screening length respectively.

- In particular, results will be influenced by the finite total length $L_{t o t}$ of polyelectrolyte chains studied in experiments or simulations. The importance of these effects varies with the ratio of the screening length $Y$ and the contour length $X$ of the blob chain. As a consequence, they risk to mask the asymptotic $Y$ dependence of observables such as the electrostatic persistence length, if they are evaluated for chains with fixed $L_{\text {tot }}$.

Quite obviously, the discrimination between the two scaling pictures requires the investigation of chains whose $e f$ fective length $X$ is as large as possible. In addition one should rely on those observables and data representations which are most sensitive to the differences between the theories and least sensitive to the omitted constants, corrections and crossovers. In the following we discuss the analysis of data for internal distances and for the tangent correlation function. In particular, we will argue that it is relatively easy to discard the KMBJ picture using simple scaling plots, while the verification of some of the predictions by the OSFKK theory requires astronomical chain lengths.

Consider first the scaling predictions Eqs. (8) to (13) for the mean square internal distances at reduced screening lengths $Y>1$. Describing the GPVB crossover to the blob pole within the chain-under-tension model [8, 32], Eqs. (8) and (9) can be combined as $\left\langle r^{2}\right\rangle / \xi^{2}=X+X^{2}$. Taking this into account, the KMBJ theory suggests that all data points should collapse when plotted in the following manner as a function of the KMBJ persistence length:

$$
\frac{\left\langle r^{2}(Z=X / Y)\right\rangle / \xi^{2}-X}{X^{2}} \simeq \begin{cases}1 & \text { for } Z<1 \\ Z^{-4 / 5} & \text { for } Z>1\end{cases}
$$

In contrast, the OSFKK theory predicts data collapse, if the segment lengths are rescaled with the OSFKK persistence length $Y^{2}$, and a breakdown of scaling for segment lengths approaching the Flory length $Y^{4}$ :

$$
\frac{\left\langle r^{2}\left(Z=X / Y^{2}\right)\right\rangle / \xi^{2}-X}{X^{2}} \simeq \begin{cases}1 & \text { for } Z<1 \\ Z^{-1} & \text { for } 1<Z<Y^{2} \\ Y^{-2 / 5} Z^{-4 / 5} & \text { for } Y^{2}<Z\end{cases}
$$

The predictions of the two scaling theories differ most strongly for chain radii along the $Y=X^{1 / 2} \mathrm{KK}_{1}$ line for segment lengths equal to the OSFKK persistence length. In the KMBJ theory, this line is already deep in the SAW regime. Eqs. (12) and (13) imply

$$
\frac{\left\langle r^{2}\left(X, Y=X^{1 / 2}\right)\right\rangle}{X \xi^{2}} \simeq \begin{cases}X^{3 / 5} & (\mathrm{KMBJ}) \\ X & (\mathrm{OSFKK})\end{cases}
$$

Thus the ratio $\left\langle r^{2}\right\rangle_{O S F K K} /\left\langle r^{2}\right\rangle_{K M B J}=X^{2 / 5}$ is fairly small and $X=10^{5 / 2}$ blobs are required for this ratio to 


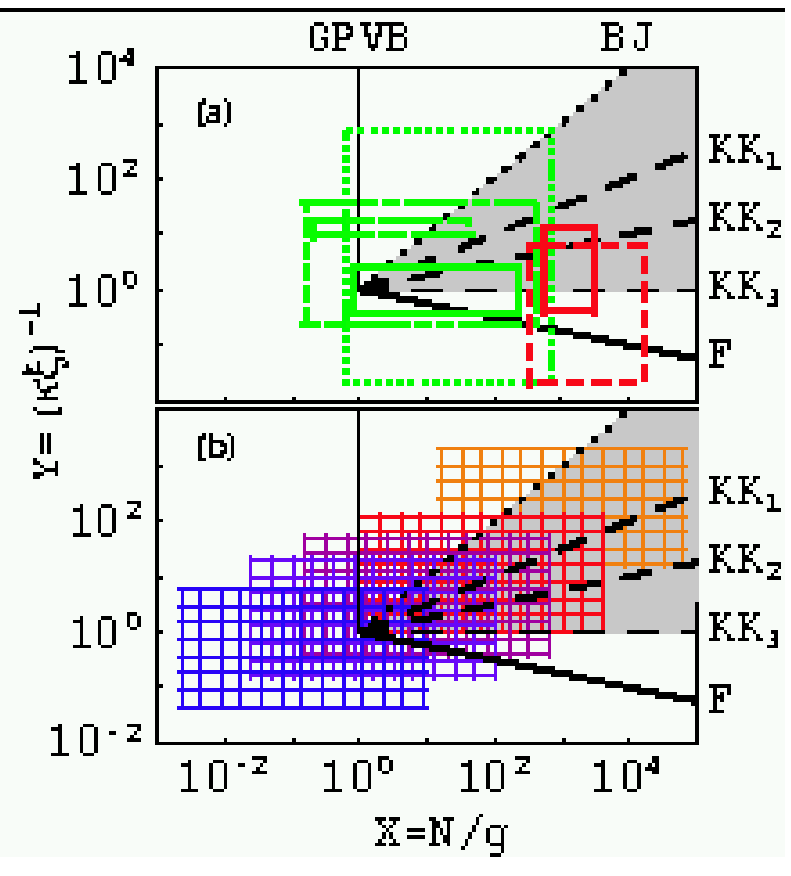

Fig. 2. Areas of the conformation diagram Fig. If for which there are experimental and simulation data available. In (a) the green lines indicate the parameter ranges investigated in previous numerical studies: Barrat and Boyer [32] (- - Seidel 33 (- Jönsson et al. [34, 28 $(\cdots \cdots)$, Micka and Kremer [27] (- - ). Experiments (shown in red) have access to longer chains, but the reduced screening length $Y$ are typically smaller than ten: Reed et al. 23] (-- ), Beer et al. 26] (-). The colored grids in (b) denote the parameter ranges covered by different sets of our MC simulations. Note that the predictions of the KMBJ and the OSFKK theory differ most strongly along the $\mathrm{KK}_{1}$ line and are identical outside of the the gray shaded area.

become of order 10. For comparision, both theories predict full extension along the KMBJ line

$$
\frac{\left\langle r^{2}(X, Y=X)\right\rangle}{X \xi^{2}} \simeq\left\{\begin{array}{l}
X(\text { KMBJ }) \\
X(\text { OSFKK })
\end{array}\right.
$$

and differ only by a factor of $\left\langle r^{2}\right\rangle_{O S F K K} /\left\langle r^{2}\right\rangle_{K M B J}=$ $X^{1 / 10}$ along the $\mathrm{KK}_{2}$ line

$$
\frac{\left\langle r^{2}\left(X, Y=X^{1 / 4}\right)\right\rangle}{X \xi^{2}} \simeq\left\{\begin{array}{l}
X^{2 / 5}(\text { KMBJ }) \\
X^{1 / 2}(\text { OSFKK })
\end{array}\right.
$$

While segment lengths of the order of $X=10^{3}$ blobs are thus sufficient to discriminate between the KMBJ and the OSFKK proposals for the electrostatic persistence lengt the requirements for resolving the additional crossovers predicted by the OSFKK theory are much higher. Consider again the $\mathrm{KK}_{2}$ line where excluded volume effects are expected to become relevant for the undulating blob chain. Eq. (13) can be rewritten in the form

$$
\frac{\left\langle r^{2}\left(Z=Y^{-4 / 5} X^{1 / 5}\right)\right\rangle}{Y^{2} X \xi^{2}} \simeq\left\{\begin{array}{l}
1 \text { for } Z<1 \\
Z \text { for } Z \geq 1
\end{array}\right.
$$

where $Z$ is the variable measuring the effective distance from the crossover line at $Z=1$. In order to identify the asymptotic behavior one needs at least data covering the interval $Z \in[0.1,10]$. Since the validity range of Eq. (19) is limited by the $\mathrm{KK}_{1}$ and $\mathrm{KK}_{3}$ lines (so that $1<Y<$ $X^{1 / 2}$ or $X^{1 / 5}>Z>X^{-1 / 5}$ ), this implies a minimum segment length of $X=10^{5}$ blobs for establishing the $\mathrm{KK}_{2}$ crossover. Similarly the $\mathrm{KK}_{3}$ crossover between Eqs. (11) and (13) at $Y=1$ becomes relevant for chains of $X=10^{10}$ blobs!

The mean-square internal distances and the tangent correlation function (TCF) obey a Green-Kubo like relation:

$$
\left\langle\mathbf{b}_{N} \cdot \mathbf{b}_{0}\right\rangle=\frac{1}{2} \frac{d^{2}}{d N^{2}}\left\langle r^{2}(N)\right\rangle=\frac{1}{2} \frac{\xi^{2}}{g^{2}} \frac{d^{2}}{d X^{2}} \frac{\left\langle r^{2}(X)\right\rangle}{\xi^{2}}
$$

For a WLC the TCF is simply given by

$$
\langle\mathbf{b}(s) \cdot \mathbf{b}(0)\rangle=b^{2} \exp \left(-s / l_{p}\right)
$$

so that the persistence length can be read off directly from a semi-logarithmic plot. Numerical studies of polyelectrolytes 27, 28,29] have therefore often focused on this quantity in spite of two intrinsic problems: (i) the TCF is considerably more difficult to measure with the same relative precision than internal distances and (ii) the TCF is particularly sensitive to finite chain length effects (a characteristic sign is the faster than exponential decay of the TCF on length scale approaching the chain length). In contrast to the case of ordinary SAWs [35], nothing is known about the functional form of the corrections. In the following discussion we will focus on a third aspect: the sensitivity of the TCF to the neighborhood of crossover lines.

On a scaling level, the behavior of the TCF can be obtained by applying Eq. (20) to Eqs. (8) to (13). For $Y>1$ the KMBJ theory predicts

$$
\frac{g^{2}}{\xi^{2}}\langle\mathbf{b}(Z=X / Y) \cdot \mathbf{b}(0)\rangle \simeq \begin{cases}1 & \text { for } Z<1 \\ Z^{-4 / 5} & \text { for } Z>1\end{cases}
$$

Within the OSFKK theory, simple predictions can only be made for segment lengths below the persistence length and beyond the Flory length:

$$
\frac{g^{2}}{\xi^{2}}\left\langle\mathbf{b}\left(Z=X / Y^{2}\right) \cdot \mathbf{b}(0)\right\rangle \simeq \begin{cases}1 & \text { for } Z<1 \\ Y^{-2 / 5} Z^{-4 / 5} & \text { for } Y^{2}<Z\end{cases}
$$

However, since both theories are based on the analogy to a mechanical WLC, they are often associated with the much more detailed prediction

$$
\begin{aligned}
& \frac{g^{2}}{\xi^{2}}\langle\mathbf{b}(X) \cdot \mathbf{b}(0)\rangle \simeq \\
& \left\{\begin{array}{l}
\exp (-X / Y) \text { for } X<Y \quad(\text { KMBJ }) \\
\exp \left(-X / Y^{2}\right) \text { for } X<Y^{2} \quad(\text { OSFKK })
\end{array}\right.
\end{aligned}
$$


for the functional form of the decay of the tangent correlations. Measuring this quantity for DHWLC therefore seems to be the most direct way of justifying or refuting this analogy and its exploitation. In particular, numerical work 27,28, 29] has concentrated on (i) establishing the existence of a single exponential decay of the TCF over a certain range of length scales and (ii) extracting the $\kappa$-dependence of the measured decay length. In the following we will reexamine this approach by taking a closer look at Eqs. (22) and (23), since they contain additional crossovers neglected in Eq. (24).

The situation should be uncritical for the GPVB crossover where the chain-under-tension models [8,32] suggests that Eq. (24) remains valid for $X<1$. In contrast, nothing is known in detail about the way the TCF crosses over to the slow power law decay characteristic for the SAW behavior on large length scales. However, matching Eq. (24) (which only accounts for the local bending rigidity) with the asymptotic behavior in Eqs. (22) and (23) shows that the tangent-correlation function is much more sensitive to excluded volume effects than the chain radii. This is most obvious for the OSFKK theory where the two limits match close to the OSFKK persistence length $X=Y^{2}$ instead of the Flory length $X=Y^{4}$. While the scaling of the TCF with the OSFKK persistence length should start to break down around $X / Y^{2} \approx 1$, one can nevertheless expect Eq. (24) to hold up to this point. In the case of the KMBJ theory the situation is quite different, since the persistence length and the Flory length coincide. As a consequence, Eq. (24) effectively breaks down as soon as the tangent-correlation function starts to deviate from one. On the other hand, in the absence of other relevant length scales the TCF should scale with the KMBJ persistence length for arbitrary segment length!

In our opinion, these arguments shed some doubts on attempts to identify the electrostatic presistence length which are based too closely on Eq. (24). Scaling plots testing Eqs. (22) and (23) may offer a simpler and safer alternative.

\section{Simulation Model, Method and Parameters}

As already mentioned in section 2, we model the polymers as freely jointed chains (FJC) with unit charges $q=$ 1 at each joint. Lengths were measured in units of the bond length $b$. We varied the the Bjerrum length $l_{B}=$ $4^{2}, 1,1 / 4^{2}, 1 / 16^{2}, 1 / 100^{2} b$ and the screening length $\kappa^{-1}=$ $1,2,4,8,16,32,64,128 \mathrm{~b}$. As in our previous study on polyampholytes [36], the chains have a length of up to $N=$ 4096 monomers.

- Since we study the conformations of isolated chains, we employ the efficient technique of pivot rotations due to Sokal et al. 37, 34]. We use two types of pivot moves: Either we rotate the part between the free end of the chain and a randomly selected monomer around an axis, defined by the bond between this monomer and its nearest neighbor; or we rotate a segment between two randomly selected monomers around an axis joining them. The latter provides better efficiency in the case of a stretched chain with large excess charge. One MC step consists of $N$ attempted rotations at random positions along the chain. Chain conformations are stored at intervals of 8-32 MC steps. For each parameter set we simulate 8 independent Markov chains in parallel. We typically store $8 \times 60$ conformations representing a total of $1.5 \times 10^{7}$ attempted rotations for our longest chains.

- Instead of the slower procedure of Stellman and Gans 38 which corrects the accumulating numeric error in offlattice implementations of the pivot algorithm, we regularly reconstruct the chains with the correct bond length.

- For calculating the long-range electrostatic interactions we use a direct summation whereby the energy of the system is obtained by direct counting of all the pair energies of the beads. This method is still efficient for macromolecules of up to few thousand monomers. The $\mathrm{DH}$ potential is tabulated in two arrays for short and long distances respectively.

- For better efficiency starting configurations of the chains are created by means of the configurational biased[39] MC method although one should keep in mind that due to the long range interactions the first part of the newly grown chain does not experience the cumulative field of the rest of the chain and a number of rotational moves are still needed before the chain is well equilibrated. Measurements are performed and conformations stored only after the chain end-to-end distances are well equilibrated.

- Since statistics is gathered both with respect to chain conformations as well as to different Bjerrum length $l_{B}$ and screening length $\kappa^{-1}$, we use a simple parallelization where different processors of a CrayT3E supercomputer perform independent simulation of single chains. The total CPU time used for this project is of the order of $1.510^{5}$ single processor hours.

The simulation parameters translate into our blob units as

$$
\begin{aligned}
& g=\left(\frac{b}{l_{B}}\right)^{2 / 3} \quad(g>1) \\
& \xi=b\left(\frac{b}{l_{B}}\right)^{1 / 3} .
\end{aligned}
$$

and

$$
\begin{aligned}
& g=\frac{b}{l_{B}} \quad(g \leq 1) \\
& \xi=b \frac{b}{l_{B}} .
\end{aligned}
$$

where we now use the number $g$ of monomers per blob instead of the corresponding contour length $l_{g}=b g$.

Fig. (2) shows where our own data are located within the $X Y$-conformation space. The effective chain and screening lengths studied cover a range of seven and five orders 


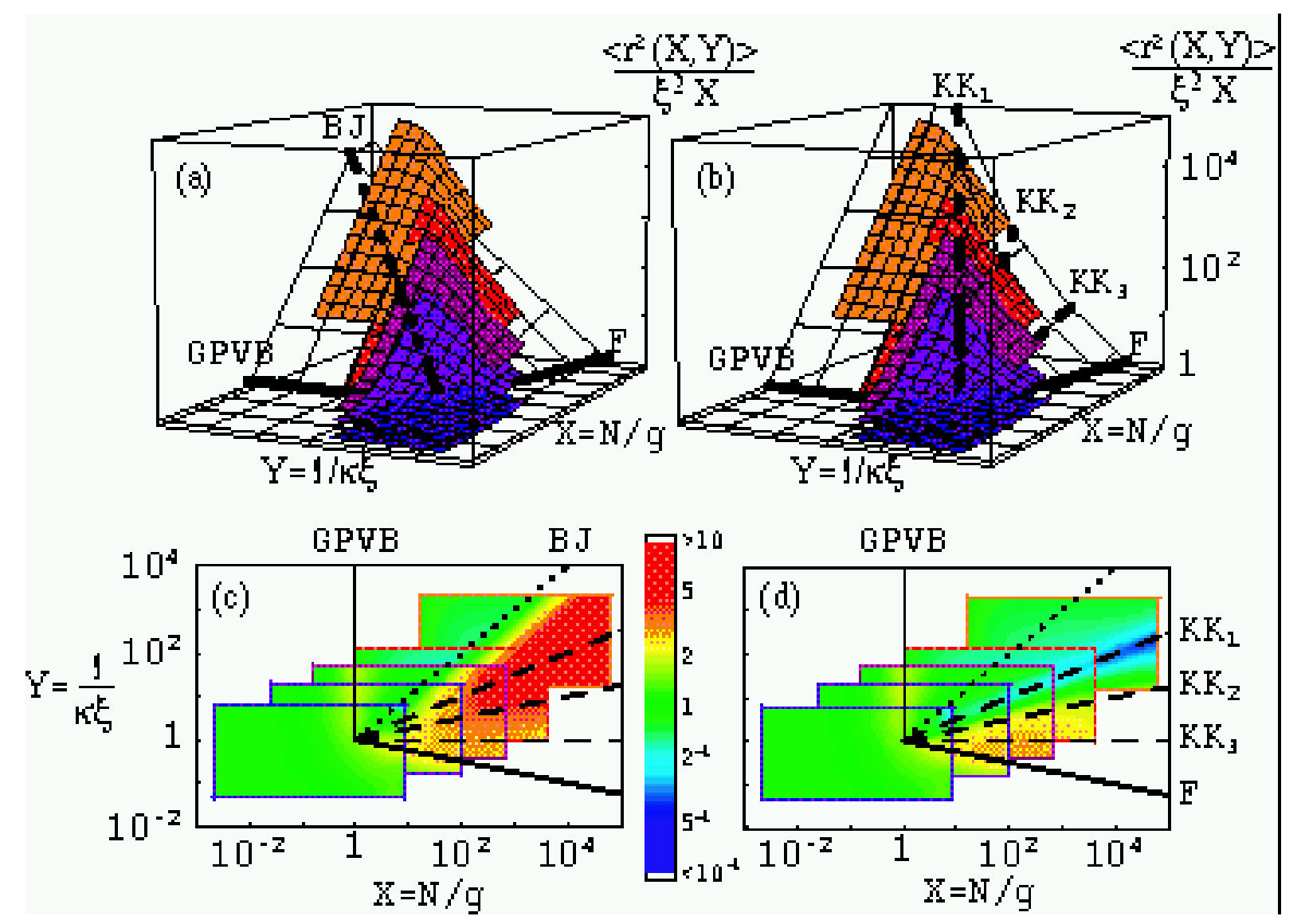

Fig. 3. Comparison of measured internal distances $\left\langle r^{2}(X, Y)\right\rangle / \xi^{2}$ to the predictions of the KMBJ ((a) and (c)) and OSFKK ((b) and (d)) scaling theories. In the top row we show log-log-log representations where all distances are normalized to the undisturbed random walk. The colored areas were generated by interpolation between the results of all simulations for a given coupling strength. The supporting grids and the crossover lines show the two sets of scaling predictions Eqs. (8) to (13) as an extensions of Fig. (1) to three dimensions. In the plots of the bottom row the colors indicate the ratios $\left\langle r^{2}(X, Y)\right\rangle /\left\langle r^{2}\right\rangle_{O S F K K}$ and $\left\langle r^{2}(X, Y)\right\rangle /\left\langle r^{2}\right\rangle_{K M B J}$ respectively.

of magnitude respectively. The reduced mean square internal distances vary over ten orders of magnitude. Along the $\mathrm{KK}_{1}$ line our data extend on a logarithmic scale about a factor of two further into the asymptotic regime than previous studies. While this allows us to discriminate between the KMBJ and the OSFKK predictions for the electrostatic presistence length, our chains are still too short to resolve the different RW and SAW regimes predicted by the OSFKK theory.

Note, that only by studying strongly stretched chains we are able to push the effective chain length $X$ close to $10^{5}$ and that our unified description of strongly and weakly charged flexible polyelectrolytes needs to be confirmed by the data analysis. To facilitate the comparison, all figures make use of the same color code to indicate data obtained for a particular coupling strength $l_{B} / b$ ranging from blue for $g=10000^{2 / 3} \approx 470$ over different shades of violet for $g=256^{2 / 3} \approx 40$ and $g=16^{2 / 3} \approx 6.4$ to red for $g=1$ and orange for $g=1 / 16$. The first three systems can safely be regarded as Gaussian chains, while the last two are at and beyond the crossover to the strong stretching regime.

\section{Results}

In the data analysis we mainly concentrate on identifying the scaling behavior: (i) Do different data sets overlap when rescaled according to our extension Eq. (5) of the definition of the electrostatic blob? (ii) How do the results of our simulations compare to the predictions of the KMBJ and OSFKK scaling theories? In terms of observables we start by presenting data for internal distances averaged along our chains of total length $N=4096$. In the second part, we discuss results for the tangent correlation function. While the TCF was also averaged along the chain, we only take into account distances up to half the chain length in order to reduce finite-chain length effects [35]. Except for the most weakly charged system, the chain extensions are much larger than the screening length, so that we do not expect finite chain length effects to be very important. At the end, we briefly present results for shorter chain lengths.

Figs. 3(a) and (b) are three-dimensional log-log-log plots giving an overview of all data. We show reduced internal distances $\left\langle r^{2}(X, Y)\right\rangle /\left((N / g) \xi^{2}\right)$ normalized to the 


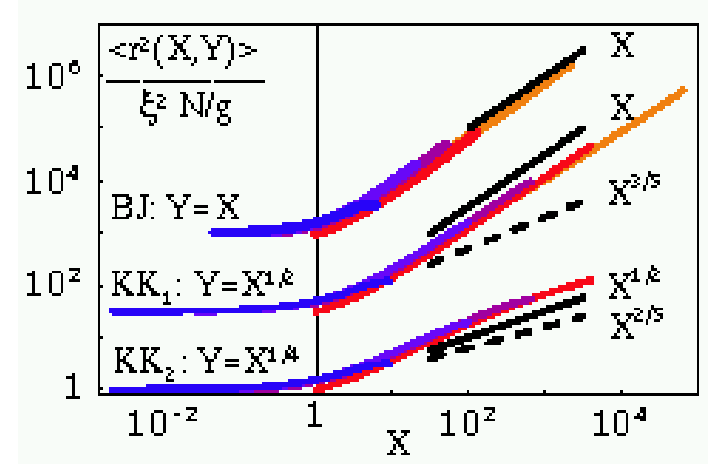

Fig. 4. Extension of chain segments along the BJ, $\mathrm{KK}_{1}$, and $\mathrm{KK}_{2}$ crossover lines in comparison to the predictions of the OSFKK (solid line) and the KMBJ (dashed line) scaling theories (see Eqs. (17), (16), and (18); note that we have not used additional prefactors for this comparison). Results for different coupling constants are shifted by factors of $\sqrt{1000}$.

size of the undisturbed random walk as a function of the reduced chain and screening lengths Eqs. (6) and (7) respectively. Results for different coupling constants are combined into colored surfaces, while the supporting grids show the two sets of scaling predictions Eqs. (8) to (13) as an extensions of Fig. 1 to three dimensions.

The complementary Figs. 3(c) and (d) show the ratios $\left\langle r^{2}(X, Y)\right\rangle /\left\langle r^{2}(X, Y)\right\rangle_{K M B J}$ and $\left\langle r^{2}(X, Y)\right\rangle /\left\langle r^{2}(X, Y)\right\rangle_{O S F K I}$ of the interpolated simulation results to the scaling predictions in a color coding where green, red and blue indicate agreement, under- and overestimation by a factor of three or more respectively. The advantage of this representation is the localization of deviations in our schematic map of the parameter space.

Qualitatively, the interpretation of Fig. 3 seems clear. There are neither indications for a failure of the blob scaling nor for significant deviations from the predictions of the OSFKK theory. (We emphasize again that we have neglected all numerical prefactors and that Eqs. (8) to (13) treat crossovers in the crudest manner). In particular, there is no evidence that the chains start to bend on length scales comparable to the screening length as predicted by KMBJ . In the relevant part of conformation space, the KMBJ theory systematically underestimates the observed chain extensions.

Nevertheless, Fig. 3 could be misleading, since the rejection of the KMBJ theory is mainly based on data falling into the strong-stretching regime, while the theory is meant to apply to weakly stretched Gaussian chains. Thus so far our conclusions rest on the assumption that the extension Eq. (5) of the blob scaling to strongly charged chains can be used to extrapolate the behavior of weakly charged systems to segment lengths inaccessible by simulation. How well this assumption is fulfilled is hard to judge from Fig. 3. Definite conclusions require a more detailed analysis.

Fig. (1) presents chain radii measured along the BJ, $\mathrm{KK}_{1}$ and $\mathrm{KK}_{2}$ crossover lines. The first point to note is that in all three cases we observe almost perfect scaling of
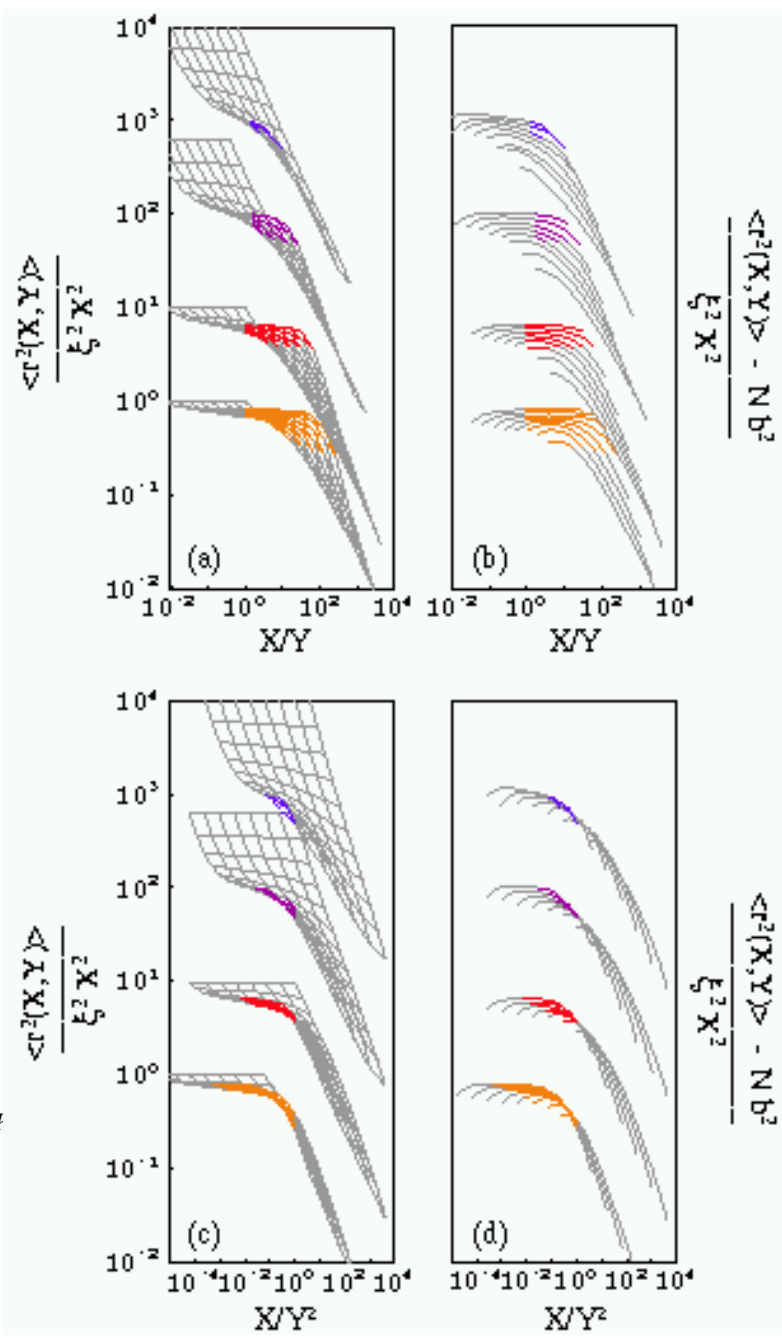

Fig. 5. Crossover scaling for internal distances versus segment length. In the top (bottom) row segment lengths $X$ are normalized to the KMBJ (OSFKK ) persistence length $Y\left(Y^{2}\right)$ respectively. Figures (a) and (c) on the left-hand side show internal distances $\left\langle r^{2}(X, Y)\right\rangle$ normalized to the mean-square extension $\xi^{2} X^{2}$ of the blob pole. The grid is the same as in Fig. 2(b) and shows const $-X$ and const $-Y$ lines. Figures (b) and (d) on the right-hand side are inspired by the chainunder-tension model for Gaussian chains Eqs. (14) and (15). Only const $-X$ lines are shown. Data points falling into the range $1<Y<X<Y^{2}$ are marked using the color code indicating the coupling strength. Results for different coupling constants are shifted by factors of ten.

data obtained for different coupling constants. Eqs. (16) to $(18)$ predict simple crossovers at the blob size around $X=1$. In agreement with both scaling pictures, we observe stretched blob chains along the BJ line. The most important set of data are the radii measured along the $\mathrm{KK}_{1}$ line for chains with a contour length $X=Y^{2}$ equal to the OSFKK persistence length. In agreement with the OSFKK theory we find a simple crossover around $X=1$ to stretched blob chains. Contrary to the predictions of the KMBJ theory the radii are essentially identical to those 

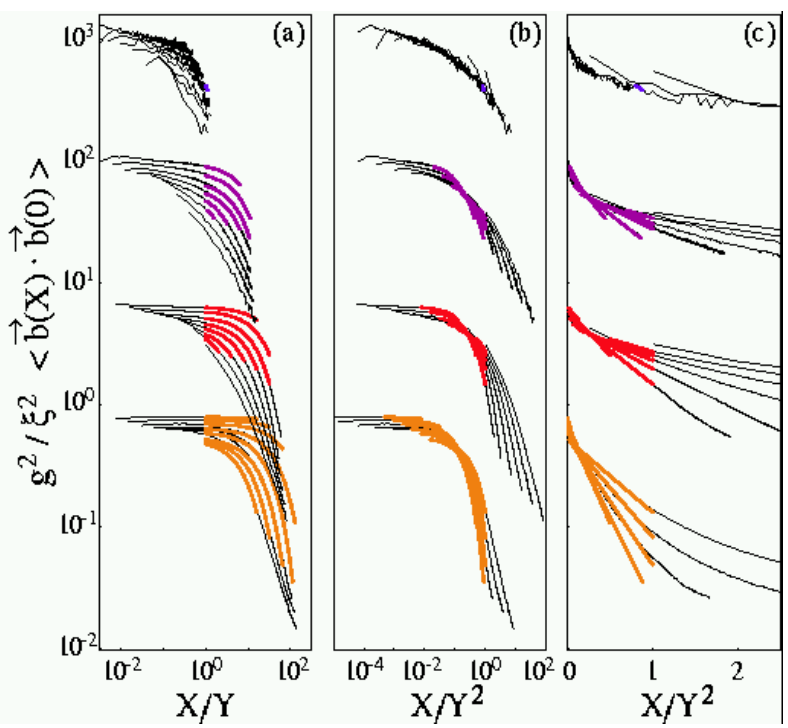

Fig. 6. Scaled tangent correlation functions. Results for different coupling constants are shifted by factors of ten. The colored lines mark the results of our fits to a simple exponential decay in the range $Y<X<Y^{2}$. (a) log-log plot using KMBJ scaling, (b) log-log plot using OSFKK scaling, (c) semi-log plot using OSFKK scaling.

observed along the BJ line and do not show SAW behavior. In particular, the asymptotic slope predicted by the OSFKK theory is already observable for weakly charged chains to which the KMBJ theory can be applied directly. The last set of data is taken along the $\mathrm{KK}_{2}$ line which marks the onset of excluded volume effects in the OSFKK theory. Here our results are consistent with the predictions of both theories. This observation is in agreement with the estimate of a minimum segment length of $X=10^{10}$ blobs for the difference to become relevant (see Eq. (18)).

The screening length dependence of the effective bending rigidity of the blob chain can also be determined from crossover scaling of internal distances normalized to the size of the stretched blob pole (Fig. 5). The disadvantage of plotting $\left\langle r^{2}(X, Y)\right\rangle /\left(\xi^{2} X^{2}\right)$ directly (Figs. . (a) and (c)) is the occurence of a $1 / X$ divergence of results for segment lengths smaller than the blob size. Correcting for this in the manner suggested by the chain-under-tension model (Eqs. (14) and (15)) as in Figs. 5 (b) and (d) largely eliminates effects due to the GPVB crossover, but introduces some artifacts for small $N$ where $\left\langle r^{2}(N=1)\right\rangle-b^{2} \equiv 0$ for a FJC. In agreement with our previous results and independently of the coupling constant we observe extremely poor scaling when the data are plotted as a function of the ratio $X / Y$ of chain length over KMBJ persistence length. In constrast, the data superimpose considerably better, if the OSFKK scaling is used as in the corresponding Figs. 5(c) and (d). In particular, Fig. 5 eliminates the possibility of an electrostatic persistence length scaling like $\kappa^{-1}$ but with an unusually large prefactor.

Similar conclusions can be drawn from an analysis of the tangent-correlation function (Fig. 6). In fact, our dis-

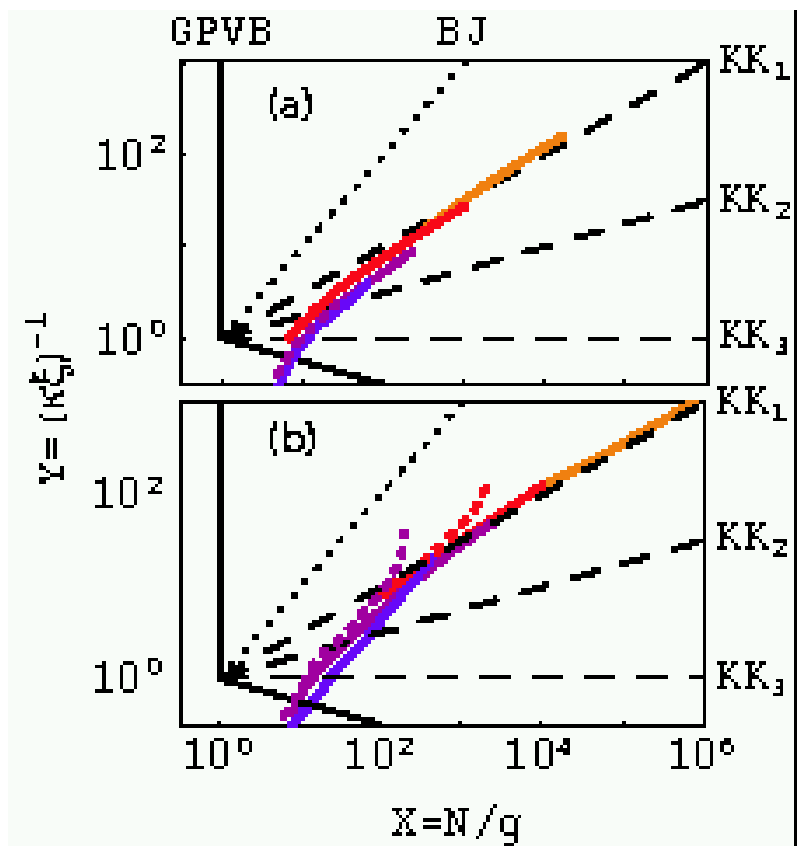

Fig. 7. Location of apparent electrostatic persistence lengths $l_{e, a p p}$ in our schematic map of the $X Y$ parameter space. We show results for the reduced crossover distance $X_{c d}=l_{c d} / \xi$ (a) and the reduced orientational correlation length $X_{o c}=l_{o c} \xi(\mathrm{b})$ for chains of length $N_{t o t}=4096$ (solid lines) and $N_{t o t}=256$ (dashed lines). In the first case, we compare $\left\langle r^{2}\right\rangle(X, Y)$ to the size of the stretched blob pole Eq. 9 and define $X_{c d}=l_{c d} / \xi$ implicitely via $\left\langle r^{2}\right\rangle\left(X_{c d}, Y\right) \equiv \xi^{2} X_{c d}^{2} / 3$. The results for $X_{o c}$ presented in (b) are decay lengths extracted from fits of TCFs to simple exponentials. For the fits we used data from the segment length interval $X<Y, \frac{1}{2} N_{\text {tot }} / g$. However, for $Y<10$ the decay of the TCFs ceases to be well described by a simple exponential (see also Fig. 6). Being strongly depend on the data range selected for the fits (data not shown, the values presented in (b) for $Y<10$ thus have to be taken with a grain of salt. We note that the results for datasets with different coupling constant scale quite well and that there is good qualitative agreement between the two methods. The results nicely follow the OSFKK prediction for reduced screening lengths $Y>10$, but are strongly influenced by excluded volume effects for smaller values of $Y$. In particular, the extracted persistence lengths systematically exceed the OSFKK estimate. With respect to finite chain length effects the first method turns out to be more robust than the second.

cussion in section 3 shows that Figs. 司 (b,d) and the log-log plots in Figs. $6(\mathrm{a}, \mathrm{b})$ are directly comparable. Fig. 6 (c) shows the same data in the semi-logarithmic representation commonly used to identify a simple exponential decay of the correlation function. Clearly, the OSFKK scaling does not work perfectly up to the OSFKK persistence length, but, at least qualitatively, we observe the expected slow-down of the decay of the correlations.

The discrepancy between our conclusion, $l_{e} \sim \kappa^{-y}$ with $y=2$, and the results of previous numerical and experimental investigations, $y \ll 2$, can be traced back to the definition of the electrostatic persistence length. So far 
we have used an indirect method (scaling plots of segment radii and TCFs). More direct methods usually proceed by (i) defining an apparant electrostatic persistence length $l_{e, a p p}$, (ii) calculating $l_{e, a p p}$ for numerical or experimental data, (iii) plotting $l_{e, a p p} / \xi$ as a function of the reduced screening length $Y$ (Fig. đ), and (tentatively) (iv) extracting effective values for $y$. Suitable definitions for $l_{e, a p p}$ were recently reviewed by Ullner and Woodward 29]. Fig. (7locates our results for the "orientational correlation length" $l_{o c}$ and the "crossover distance" $l_{c d}$ in our map of conformation space. The first length is defined as the decay length of a simple exponential fitted to the TCF while the second tries to identify the crossover from the blob pole Eq. (9) to an undulating blob chain Eqs. (12) or (13).

Clearly, the extracted length scales can only be identified with the electrostatic persistence length $l_{e}$ as long as $l_{e}$ is well separated from other relevant length scales, i.e. for sufficiently large chain and screening lengths. Fig. 7 shows that our results closely follow the $\mathrm{KK}_{1}$ line for $N=4096$ and $Y>10$. However, the violation of either of the two conditions leads to deviations. Relative to the OSFKK prediction the extracted persistence lengths

increase for small reduced screening lengths due to excluded volume effects and

decrease in the opposite limit due to finite chain length effects which are particularly strong for the TCF and quantities related to it.

Depending on the definition of $l_{e, a p p}$ and the range of chain and screening lengths studied, the combination of these two effects can lead to the observation of effective exponent $l_{e, a p p} \sim \kappa^{-y}$ which are much smaller than $y=2$. However, since the weak $\kappa$ dependence of $l_{e, a p p}$ is an artifact of the definition of the quantity, there seems to be no contradition to the OSFKK theory. Attempts along these lines [27, 28, 29] therefore risk to create more confusion than insight as long as $l_{e, a p p}$ is not defined within a theoretical framework which explicitly accounts for excluded volume effects [23].

\section{Discussion}

In this paper we have combined a scaling analysis of the conformational properties of intrinsically flexible polyelectrolytes with Debye-Hückel interactions with extensive Mon Carlo simulations of isolated chains. Our study was focused on the controversial case of polyelectrolytes beyond the OSF limit, i.e. to the case where the electrostatic screening length $\kappa^{-1}$ exceeds the bare persistence length of the polymers in the absence of electrostatic interactions.

Our main result is the refutation of theories [5, 13] predicting an electrostatic persistence length scaling as $\kappa^{-1}$. In contrast, we have observed no significant deviations from the scenario proposed by Khokhlov and Khachaturian [7] who combined the idea by de Gennes et al. [8] of a stretched chain of polyelectrolyte blobs with the OdijkSkolnick-Fixman theory of the electrostatic persistence length [3, 4] and the electrostatically excluded volume [6, 30] between chain segments. Our results suggest that it is indeed possible to understand DHWLC by considering a hierarchy of effects due to interactions between different classes of monomer pairs:

Stretching due to the (effectively unscreened) Coulomb repulsion between neighboring monomers into a chain of blobs which has a finite

Bending rigidity due to the screening of interactions between monomers with a distance larger than $g /(\kappa \xi)$ along the chain. As a consequence, the blob chain remains straight up the electrostatic persistence length $l_{e}=\kappa^{-2} / \xi$. Beyond $l_{e}$ the chain behaves like a random walk, before

Swelling due the electrostatically excluded volume between chain segments with a distance larger than $l_{e}$ becomes relevant beyond the Flory length $l_{F}=\kappa^{-4} / \xi^{3}$

An interesting side result of our work is the extension of the polyelectrolyte blob scaling to the case of strongly interacting, almost fully stretched chains for which the OSF theory is known to work [6]. This extension was done in the logic of the OSFKK theory but is incompatible with the ansatz of BJ. Its success provides strong evidence for the irrelevance of longitudinal and transverse fluctuations within the blob chain 19] and proves the KK idea almost by itself.

Clearly, scaling arguments cannot do justice to the full complexity of the problem. Omitting all numerical prefactors, the ubiquituous logarithmic corrections, finite chain length effects and, in our opinion most importantly, a refined description of the crossovers between narrow neighboring regimes, they cannot hope (and should not be expected) to describe numerical or experimental data in detail. Quite obviously, these features call for a quantitative explanation. While our numerical results can serve as benchmarks for the development of theories, it is a sobering thought that the simplest model of a single, isolated polyelectrolyte chain is still unsolved. Compared to the much better understood neutral polymers, the OSFKK theory represents the equivalent of the standard Flory argument for the excluded volume effect. Nevertheless, we believe to have shown that the OSFKK theory provides the indispensible "big picture" needed for the design and analysis of experiments and computer simulations.

\section{Acknowledgements}

We gratefully acknowledge helpful discussions with B. Dünweg, J.-F. Joanny, K. Kremer and H. Schiessel. RE is supported by an Emmy-Noether fellowship of the DFG.

\section{References}

1. J.-L. Barrat and J.-F. Joanny. Theory of polyelectrolyte solutions. Advances in Chemical Physics, 94:1-65, 1996.

2. M. Ullner. Polyelectrolyte models in theory and simulation. In S. Tripathy, J. Kumar, and H. S. Nalwa, editors, Handbook of Polyelectrolytes and Their Applications. American Scientific Publishers, Los Angeles, 2002. 
3. T. Odijk. Polyelectrolytes near the rod limit. Journal of Polymer Science: Polymer Physics Edition, 15:477-483, 1977.

4. J. Skolnick and M. Fixman. Electrostatic persistence length of a wornlike polyelectrolyte. Macromolecules, 10:944-948, 1977.

5. J.-L. Barrat and J.-F. Joanny. Persistence length of polyelectrolyte chains. Europhysics Letters, 24:333-338, 1993.

6. T. Odijk and A. C. Houwaart. On the theory of the excluded-volume effect of a polyelectrolyte in a 1-1 electrolyte solution. Journal of Polymer Science: Polymer Physics Edition, 16:627-639, 1978.

7. A. R. Khokhlov and K. A. Khachaturian. Polymer, 23:1793, 1982.

8. P. G. de Gennes, P. Pincus, R. M. Velasco, and F. Brochard. Remarks on polyelectrolyte conformation. Journal de Physique (France), 37:1461-1473, 1976.

9. M. Muthukumar. Adsorption of a polyelectrolyte chain to a charged surface. Journal of Chemical Physics, 86:72307235, 1987.

10. M. Muthukumar. Double screening in polyelectrolyte solutions: Limiting laws and crossover formulas. Journal of Chemical Physics, 105:5183-5199, 1996.

11. K. Ghosh, G. A. Carri, and M. Muthukumar. Configurational properties of a single semiflexible polyelectrolyte. Journal of Chemical Physics, 115:4367-4375, 2001.

12. W. Kuhn, O. Künzle, and A. Katchalsky. A. Helv. Chim. Acta, 31:1994, 1948.

13. B.-Y. Ha and D. Thirumalai. Electrostatic persistence length of a polyelectrolyte chain. Macromolecules, 28:577581, 1995.

14. T. A. Vilgis and J. Wilder. Polyelectrolyte networks: elasticity, swelling, and the violation of the flory-rehner hypothesis. Computational and Theoretical Polymer Science, 8:61-73, 1998.

15. J. Wilder and T. A. Vilgis. Elasticity in strongly interacting soft solids: A polyelectrolyte network. Physical Review E, 57:6865-6874, 1998.

16. T. B. Liverpool and M. Stapper. The scaling behaviour of screened polyelectrolytes. Europhysics Letters, 40:485-490, 1997.

17. T. B. Liverpool and M. Stapper. Renormalization group analysis of weakly charged polyelectrolytes. Eur. J. Phys. E, 5:359-375, 2001.

18. P. L. Hansen and R. Podgornik. Wormlike chains in the large-d limit. Journal of Chemical Physics, 114:8637-8648, 2001.

19. H. Li and T. A. Witten. Fluctuations and persistence length of charged flexible polymers. Macromolecules, 28:5921-5927, 1995.

20. B.-Y. Ha and D. Thirumalai. Persistence length of flexible polyelectrolyte chains. Journal of Chemical Physics, 110:7533-7541, 1999.

21. R. R. Netz and H. Orland. Variational theory for a single polyelectrolyte chain. Eur. J. Phys. B, 8:81-98, 1999.

22. M. Tricot. Comparison of experimental and theoretical persistence length of some polyelectrolytes at various ionic strengths. Macromolecules, 17:1698-1704, 1984.

23. W. F. Reed, S. Ghosh, G. Medjahdi, and J. Francois. Dependence of polyelectrolyte apparant persistence lengths, viscosity, and diffusion on ionic strength and linear charge density. Macromolecules, 24:6189-6198, 1991.
24. S. Förster, M. Schmidt, and M. Antonietti. Experimental and theoretical investigation of the electrostatic persistence length of flexible polyelectrolytes at various ionic strength. Journal of Physical Chemistry, 96:4008-4014, 1992.

25. S. Förster and M. Schmidt. Polyelectrolytes in solution. Advances in Polymer Science, 120:50-133, 1995.

26. M. Beer, M. Schmidt, and M. Muthukumar. The electrostatic expansion of linear polyelectrolytes: Effects of gegenions, co-ions, and hydrophobicity. Macromolecules, 30:8375-8385, 1997.

27. U. Micka and K. Kremer. Persistence length of the debyehückel model of weakly charged flexible polyelectrolyte chains. Physical Review E, 54:2653-2662, 1996.

28. M. Ullner, B. Jönsson, C. Peterson, O. Sommelius, and B. Söderberg. The electrostatic persistence length calculated from monte carlo, variational and perturbation methods. Journal of Chemical Physics, 107:1279-1287, 1997.

29. M. Ullner and C. E. Woodward. Orientational correlation function and persistence length of flexible polyelectrolytes. Macromolecules, 35:1424-1436, 2002.

30. M. Fixman and J. Skolnick. Polyelectrolyte excluded volume paradox. Macromolecules, 11:863-871, 1978.

31. M. Castelnovo, P. Sens, and J.-F. Joanny. Charge distribution on annealed polyelectrolytes. Eur. J. Phys. E, 1:115-125, 2000.

32. J.-L. Barrat and D. Boyer. Numerical study of a charged bead-spring chain. Journal de Physique II, 3:343-356, 1993.

33. C. Seidel. Polyelectrolyte simulation. Ber. Bunsenges. Phys. Chem., 100:757-763, 1996.

34. B. Jönsson, C. Peterson, and B. Söderberg. variational approach to the structure and thermodynamics of linear polyelectrolytes with coulomb and screened coulomb interactions. Journal of Physical Chemistry, 99:1251-1266, 1995.

35. L. Schafer, A. Ostendorf, and J. Hager. Scaling of the correlations among segment directions of a self-repelling polymer chain. Journal de Physique (France), 32:78757899, 1999.

36. V. Yamakov, A. Milchev, H.-J. Limbach, B. Dünweg, and R. Everaers. Conformations of random polyampholytes. Physical Review Letters, 85:4305-4308, 2000.

37. N. Madras and A. D. Sokal. J. Stat. Phys., 50:109, 1988.

38. S. D. Stellman and P. J. Gans. Macromolecules, 5:516, 1972 .

39. D. Frenkel and B. Smit. Understanding Molecular Simulation: From Algorithms to Application. Academic Press, NY, 1996. 